\title{
Empirically Verifying Metamotivational Moments: The Identification, Analysis, and Reassembly of Style Clusters
}

\author{
Kenneth M. Cramer \& Jessica Sartori \\ University of Windsor
}

\begin{abstract}
The present study aimed to identify metamotivational style clusters using (a) cluster analysis to derive four groups or clusters of respondents who completed the Motivational Style Profile, (b) ANOVA to analyze the style composition of each cluster, and (c) discriminant function analysis to reassemble the clusters based on prescribed subscale scores (telic, paratelic, negativistic, and conformist). Undergraduate volunteers from a Canadian university completed the Motivational Style Profile for partial course credit, and we then calculated their four subscale scores. Results showed the four style clusters were reliably different from each other based on the subscale mean differences; discriminant function analysis successfully returned $83 \%$ of respondents to their original cluster. Implications for the measurement of metamotivational styles are outlined, as are directions for future research.
\end{abstract}

Keywords: Reversal Theory, cluster analysis, metamotivational style

Whether we observe a loving parent soothing a crying child, a joyous reunion of military personnel with family and friends, a classroom of students engaged in a lecture, or a ruthless CEO manipulating financial markets-each is explained by that individual's unique motive at that moment in time (Apter, 1982, 1989, 2013). According to Reversal Theory, metamotivational states represent phenomenological moments in human psychology wherein a particular motive is not only experienced, but later informs and directs future thought, feeling, and action. Although these states are everchanging, moments can be experienced in similar ways over time (wherein the loving parent described above is later witnessed as a caring friend), so that the increased frequency of experiencing a particular state may suitably reveal one's style or state dominance across a long range of instances or moments. To make clear the fine distinction between states (momentary phenomenological experiences) and styles (frequent or dominant momentary experiences), we begin with a discussion of how we measure metamotivational states; this too will offer a useful historical backdrop to the investigation. Ultimately we aim to empirically identify, analyze, and reassemble metamotivational styles using cluster analysis-a statistical technique that groups together similar entities; we propose that those similarities can be observed in the com-

Kenneth M. Cramer, Department of Psychology, University of Windsor; Jessica Sartori, Department of Psychology, University of Windsor.

Correspondence concerning this article should be addressed to Kenneth M. Cramer at kcramer@uwindsor.ca mon responses that individuals express in their dominant everyday style of behavior.

Measurement of Metamotivational States. Reversal Theory posits that human experience can be best modeled using the moment-to-moment experience of a finite set of motives that inform and direct future action, feeling, and thinking. Those motives can be divided into four opposing pairs, rendering eight metamotivational states: Telic-Paratelic, Conformist-Negativistic, Autic-Alloic, and Sympathy-Mastery (Apter, 1989). For simplicity and statistical manageability (given the modest but still adequate sample size), we investigated only the first two pairs of states. The telic or serious state is aimed at achievement and may be observed when one is goal-oriented, whereas the paratelic or fun-loving state is aimed at enjoyment and may be observed when one finds pleasure in the moment, for example, while exploring an open-world video game. The conformist or compliant state may be observed when one observes and follows the rules or expectations within a given situation, whereas the negativistic or rebellious (nonconformist) state may be observed when one aims to break those known rules. In the course of a day, individuals will routinely reverse among these paired motives to satisfy their moment-to-moment motivational intents. The saying, "all work and no play makes Jack a dull boy," reminds us that too much time spent in the telic, serious-minded state will on the one hand assuredly satisfy those achievement motives until they become saturated, leaving us on the other hand motivated to seek pleasure and recreation-in short, to "recharge our engines" so as to be successful at our next task.

The measurement of metamotivational states has been (and still remains) a challenging enterprise. Indeed, it has 
been argued that the very act of measuring a state in situ can change the very state itself-reversing an individual from a fun-loving paratelic moment spent at their child's concert to a serious-minded telic state by responding to a metamotivational survey (Cramer, 2013; Robins, Hendin, \& Trzesniewski, 2001). However, casting that matter aside, several avenues exist toward the assessment of an individual's current motivational state. One rather straightforward approach is the use of home-grown single-item measures, as Cramer and Lafrieniere (2015) demonstrated in their assessment of telic vs. paratelic states in the course of an 80minute university lecture. Alternatively, single items can be sampled from a telic-paratelic state instrument (O'Connell $\&$ Calhoun, 2001). As such, one may ask an individual at a given moment to select between two forced-choice options, whether they (a) feel more playful or serious-minded, (b) want to be more or less energetic, (c) want peace/quiet or more adventure, or (d) want to accomplish a goal or have more fun. The dialogue continues on the nature of singleitem vs. multiple-item instruments whose aim is to maximize content validity without augmenting respondent fatigue (Robins et al., 2001; Robins, Trzesniewski, Tracy, Gosling, \& Potter, 2002).

The 1980s and 1990s saw the development of various reversal theory measures that tapped the experience of specific states, including the Telic State Measure (Svebak \& Murgatroyd, 1985), the Negativism Dominance Scale (McDermott, 1988), the Telic/Paratelic State Instrument (O'Connell \& Calhoun, 2001), and the Autic Mastery-Sympathy State Measure (O'Connell \& Brooks, 1997). More recent attempts have sought to enhance accuracy while keeping the tools brief in an effort to reduce reactivity, as Desselles, Murphy, and Theys (2014) showed in their development of the Reversal Theory State Measure. This instrument, designed to assess all eight metamotivational states, comes in three forms: a 3-item "bundled" version, a 12-item logical branched version, and an 18-item full version. Whereas virtues and vices were identified among all three versions, the instrument stands as one of the few comprehensive measures of metamotivational states.

Measurement of Metamotivational Styles. Beyond the assessment of metamotivational states, we also can assess the recurring experience of a particular state that uncovers a pattern or style of daily motives. Motivational styles represent an increased likelihood that an individual spends their daily experience viewing life for example, as focused on achievement or fun. Personality theorists tapped a similar concept as they moved from the discussion of specific responses (like texting a friend) to habitual responses (like often texting friends; Eysenck \& Eysenck, 1985). The individual too may be aware of a markedly greater proportion of time spent in the telic vs. paratelic state (dominance); this adds salience to the more oft-used motive, much as Allport described personality using the notion of a cardinal trait (Allport \& Odbert, 1936).

So too, we must consider the measurement of metamotivational styles, whose psychometric assessment gained more solid footing with the advent of the Motivational Style Profile (MSP, Apter, Mallows, \& Williams, 1998), a 70-item forcedchoice inventory that measured both the dominance and salience of various properties of the phenomenological experience. In terms of our present focus on the telic/paratelic and conformist/negativistic state experience, their initial findings showed mixed results concerning internal consistencies, wherein reliability estimates using the Kuder-Richardson20-test (KR-20) ranged from a high of .83 for negativistic to a low of .53 for conformist. Later studies similarly highlighted these underwhelming reliability estimates in the assessment of the conformist style (Cramer, Lafreniere, \& Ianni, 2015; Lafreniere \& Cramer, 2006). Why the reliability issue continues to dog reversal theory is unclear. On the one hand, it is tenable that the measurement of ever-changing states would present various psychometric challenges (likely unsatisfied by typical Cronbach tenets in classical psychometric theory); on the other hand, these arguments are less defensible in the assessment of aggregate motivational styles (based on both dominance and salience). Another empirical approach to confirm style reliability is thus warranted.

Present Study and Hypothesis. We propose an alternative route toward assuring confidence in the assessment of metamotivational styles. Given the internal consistency derived from the measurable cohesiveness of its derivative subscale items (Nunnally \& Bernstein, 1994), we opted to statistically reassemble items in the MSP that were correlated to form their own entity or cluster of respondents. These clusters shared a common motivational style. We hypothesized that metamotivational styles then could be successfully identified based on similar response sets from our participants. Likewise, these empirically-derived style clusters should then correspond to the original styles as prescribed in the MSP scoring manual. In other words, our style clusters should produce reliable mean differences in telic, paratelic, conformist, or negativistic subscale scores. Finally, and most notably, we should be able to effectively return each respondent to their original cluster equipped only with their MSP responses.

\section{Method}

Participants and Measures. The data, drawn from Sartori's (2004) doctoral dissertation, consisted of 160 undergraduates at a mid-sized Canadian university who agreed to participate (as recruited from the departmental research pool so as to receive partial course credit). Participants were roughly evenly split by sex, with an average age of 20.3 years $(\mathrm{SD}=1.98)$. Participants completed the Motivational Style Profile (Apter et al., 1998), a 70-item inventory to assess 14 
Table 1

Mean Differences in Four Metamotivational Styles by Style Cluster

\begin{tabular}{lrrrrr}
\hline & Cluster A & Cluster B & Cluster C & Cluster D & \\
& $M(S D)$ & $M(S D)$ & $M(S D)$ & $M(S D)$ & $F^{*}$ \\
& $N=79$ & $N=31$ & $N=20$ & $N=30$ & $(3,156)$ \\
\hline Telic & $23.2 \mathrm{~b}(3.4)$ & $20.1 \mathrm{a}(3.1)$ & $19.0 \mathrm{a}(3.5)$ & $23.5 \mathrm{~b}(3.3)$ & 14.23 \\
Paratelic & $21.7 \mathrm{~b}(3.2)$ & $17.2 \mathrm{a}(2.2)$ & $24.8 \mathrm{c}(3.9)$ & $17.0 \mathrm{a}(2.7)$ & 41.93 \\
Negativistic & $13.3 \mathrm{~b}(2.5)$ & $9.90 \mathrm{a}(1.9)$ & $18.8 \mathrm{c}(3.7)$ & $8.93 \mathrm{a}(2.7)$ & 68.83 \\
Conformist & $19.7 \mathrm{~b}(3.4)$ & $18.2 \mathrm{a}(1.9)$ & $17.8 \mathrm{a}(3.1)$ & $22.5 \mathrm{c}(1.9)$ & 15.29 \\
$\%$ of Cases Correctly Classified & $90 \%$ & $70 \%$ & $75 \%$ & $73 \%$ & \\
\hline
\end{tabular}

Note. means with identical subscripts in a given row are not significantly different. $* p<.001$

dimensions (5 items each) of metamotivational experience (Likert responses range from $1=$ never to $6=$ always); however, only four dimensions were relevant to the present study: telic, paratelic, negativistic, and conformist; as such, those four subscale scores were calculated for each respondent.

\section{Results}

The analysis was divided into three stages: style cluster derivation, style cluster correlation to subscale scores, and then cluster reassembly. To begin, the 20 derivative MSP items (5 each) of the four metamotivational styles were included in a cluster analysis that combined participants with comparable responses to the MSP items. Using Ward's method of grouping similar respondents based on minimizing squared Euclidean distances (Everitt, 1993; Gordon, 1987), the cluster analysis identified four style clusters: Cluster A $(n=79)$, Cluster B $(n=31)$, Cluster C $(n=20)$, and Cluster $\mathrm{D}(n=30)$. Cluster $\mathrm{A}$, for example, represents a group of 79 participants who responded to the $20 \mathrm{MSP}$ items in a similar way, yet in a style distinct from the way the 31 Cluster B participants responded to the same items.

Having derived respondent membership to the four style clusters, we next conducted an analysis of variance (ANOVA) using the four clusters as the grouping variable, and the four subscale scores (telic, paratelic, negativistic, and conformist) as the dependent variables. We note presently a considerable range of internal consistency estimates: telic $(\mathrm{KR}-20=.612$, but could be increased to .642 with the exclusion of one item), paratelic (KR-20 $=.757)$, negativistic $(\mathrm{KR}-20=.794)$, and conformist $(\mathrm{KR}-20=.503$, but could be increased to .546 with the exclusion of one item; regardless, this final subscale remains unacceptably low by psychometric benchmarks; Nunnally \& Bernstein, 1994). Whereas all omnibus tests were significant $(p<.001)$, follow-up Student-Neuman-Keuls multiple comparison procedures revealed the source of the mean differences for each subscale (see Table 1). First, telic subscale scores were significantly lower for respondents in Clusters B and C, as compared to respondents in Clusters A and D (see Figure 1). Secondly, paratelic subscale scores were significantly lower for respon-

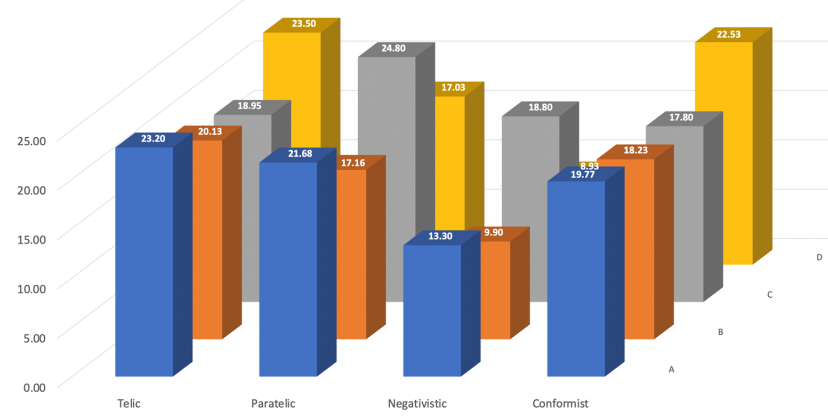

Figure 1. Telic, Paratelic, Negativistic, and Conformist Mean Differences by Cluster

dents in Clusters B and D as compared to respondents in Cluster A, whose scores were significantly lower as compared to respondents from Cluster C. Thirdly, negativistic subscale scores were significantly lower for respondents in Cluster B and D, as compared to respondents in Cluster A, whose scores were significantly lower as compared to respondents in Cluster C. Finally, conformist subscale scores were significantly lower for respondents in Clusters B and C, as compared to respondents in Cluster A, whose scores were significantly lower as compared to respondents in Cluster D. In short, Cluster A respondents may be best described as having a dominant telic style, Cluster $\mathrm{C}$ respondents as having a dominant paratelic-negativistic style, and Cluster B and Cluster D respondents as having a dominant telic-conformist style.

The final stage of the analysis was to reassemble the cluster constituents by using the four subscale scores to predict each respondent's derived cluster, using a discriminative function analysis. Much like an ANOVA in reverse, the discriminant analysis calculates one or more linear functions of the metamotivational subscales to correctly return respondents to their original cluster (thus minimizing error). As a linear function, this approach assigns specific weights to each of the four subscales so as to maximally discriminate among the four clusters. 


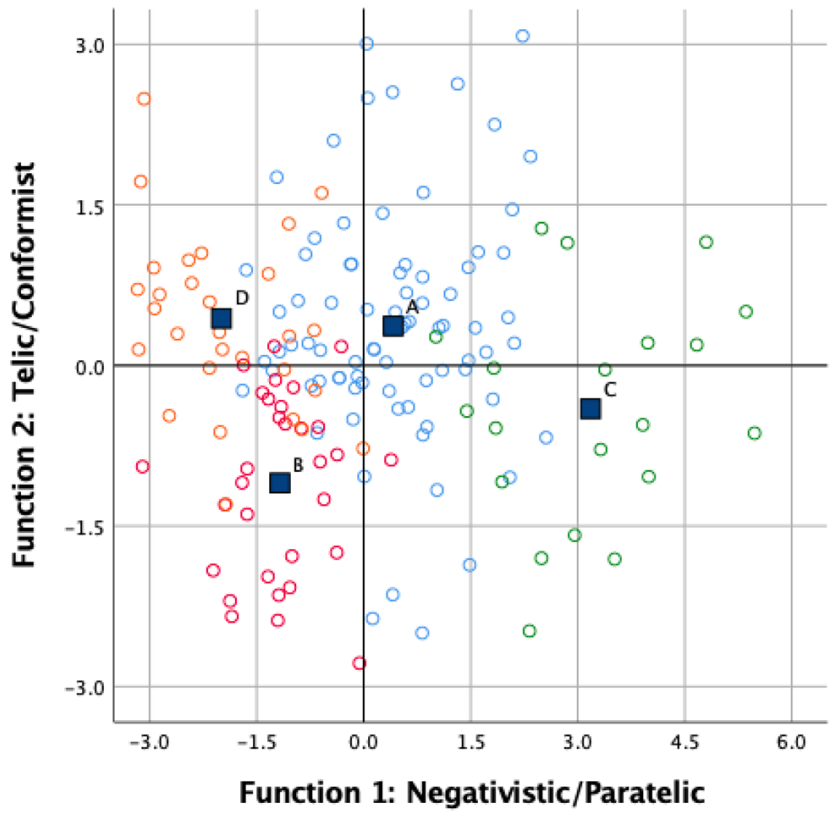

Figure 2. Territorial Map of F1: Negativistic/Paratelic vs. F2: Telic/Conformist

Based on group sizes, and with initial inclusion of all four subscales, results revealed two significant discriminant functions, explaining $98 \%$ of the variance in cluster assignment. As a linear derivative of the four subscales, the first function $\left(\chi^{2}[12]=248.45, p<.001\right)$ explained $85.0 \%$ of the variance in clusters, and consisted positively of both the negativistic $(\beta=+.738)$ and paratelic styles $(\beta=+.554)$, and negatively of both the telic $(\beta=-.140)$ and conformist styles $(\beta=-.215)$. This was the key dimension upon which the respondents' style clusters differed the most. The second function $\left(\chi^{2}[6]=57.76, p<.001\right)$ explained $12.8 \%$ of the variance in clusters, and consisted positively of each of telic $(\beta=+.752)$ and conformist styles $(\beta=+.638)$, less of the paratelic style $(\beta=.386)$, and very little of the negativistic style $(\beta=.092)$. For style clusters A through $\mathrm{D}$, both function scores and cluster centroids are plotted in Figure 2. In short, Cluster A respondents were moderate on both the Telic-Conformist and Negativistic-Paratelic styles, Cluster B respondents were low on both, Cluster $\mathrm{C}$ respondents were especially high on the Negativistic-Paratelic style, and Cluster D respondents were especially low on the NegativisticParatelic style. Finally, the reassembly of style clusters based on subscale scores yielded excellent classification rates for Cluster A $(89.9 \%$ correct assignment of respondents to the cluster based on subscale scores), and good classification rates for each of Cluster B (77.4\%), Cluster C (75.0\%), and Cluster D (73.3\%).

\section{Discussion}

As another index of psychometric validity, the present study showed that cluster analysis could successfully identify and group together respondents with similar motivational styles. Indeed, this is the first analysis of its kind to evaluate metamotivational styles according to cluster analysis. Whereas we found differences by the four style clusters according to each of telic, paratelic, negativistic, and conformist subscale scores, the discriminant function analysis showed that approximately $83 \%$ of respondents could be returned to their empirically-derived style cluster based on their $20 \mathrm{MSP}$ item responses. It is curious to note that our largest cluster (A) consisted of almost half the sample ( $n=79)$ and was typified by higher levels of the telic style, and moderate levels of paratelic, negativistic, and conformist styles. That the discriminant functions represented linear combinations or weighted degrees of styles indicates that more work is needed to see what combinations of metamotivational styles exist (and by what proportion) in the general population. In the present study, the strongest discriminator by metamotivational style was especially informed through a unique combination of all four states: higher scores indicated higher dominance of both negativistic and paratelic styles, and the inverse of both conformist and telic styles. The second function (arguably less explanatory) combined both telic and conformist styles (with little drawn from both paratelic and negativistic styles).

The present study sought a unique avenue to understanding consistency in style assessment, using an independently derived means to grouping similar entities-in this case, those respondents who share a common metamotivational style profile. That is, the present study showed that an empiricallydriven search for common motivational styles could successfully identify like-styled respondents based on their 20 MSP answers; and then accurately return $83 \%$ of them to their original style cluster based on subscale scores. Experimental research should next be carried out, employing a manipulation to induce either a telic or paratelic state, and see if cluster analysis can successfully identify randomly-assigned group. One example might involve a driving simulator, where a participant is completing the virtual course while texting, only to be confronted by an on-screen emergency (reversing them from a paratelic to telic state).

To further consider reversal theory and its marriage to cluster analysis on the broader stage is to invite a wider conversation concerning falsification and hypothesis testing. That is, reversal theory would certainly not advocate grouping respondents into clusters or categories given the fluid nature of phenomenological experience. There remains however a tradition within the empirical arena to rightly compare MSP dominances on a host of criteria (Apter et al., 1998; Cramer \& Lafreniere, 2015; Lafreniere \& Cramer, 2006). As such, we believe that cluster analysis can hope to offer rever- 
sal theory a wealth of empirical currency. Some of those fruitful directions are outlined below.

Outside the microscope of empirical hypothesis testing, we need to explore how the concept of metamotivational clusters might offer a rich interpretation of the human experience in real-world contexts, such as team building, self-development workshops, customer relations, leadership, marital therapy, and classroom interaction-domains chiefly defined by interpersonal management and accommodation. This then becomes a field rich in research possibilities; that is, what is the interpersonal dynamic within a group of people where some share a motivational intention with others? Presumably, those within a common motivational style cluster should work together more closely (even empathize and cooperate more), but struggle to accommodate those out-group members in alternative (even opposing) motivational style clusters. Future research would do well to further explore these avenues.

Implications from the present analysis are vast since it shows that consistencies in the style by which people view the world are replicable via several empirical means. We further encourage researchers to expand the analysis to all eight metamotivational states in an effort to extend the validity of the analysis of metamotivational style assessment; this of course would invite a much larger sample for analysis. Similarly, clusters could be so derived as to evaluate the tenability (and replicability) of the four somatic vs. four transactional dimensions. Future research would do well to replicate this methodology and design on the Desselles et al. (2014) trifecta of state measures, namely the 3-item bundled, the 12 -item branched, and the 18-item full inventory of metamotivational states.

\section{References}

Allport, G. W., \& Odbert, H. S. (1936). Psychological monographs, 47, i-171

Apter, M. J. (1982). The experience of motivation: The theory of psychological reversals. London, UK: Academic Press.

Apter, M. J. (1989). Reversal Theory: Motivation, emotion, and personality. London, UK: Routledge.

Apter, M. J. (2013). Developing Reversal Theory: Some suggestions for future research. Journal of Motivation, Emotion, and Personality, 1, 1-8.

Apter, M. J., Mallows, R., \& Williams, S. (1998). Development of the Motivational Style Profile. Personality and Individual Differences, 24, 7-18.

Cramer, K. M. (2013). Six criteria of a viable theory: Putting Reversal Theory to the test. Journal of Motivation, Emotion, and Personality, 1, 9-16.
Cramer, K. M., \& Lafreniere, K. L. (2015). Lecture engagement and metamotivational states: Tracking and intervention. Journal of Motivation, Emotion, and Personality, 4, 9-14.

Eysenck, H. J., \& Eysenck, M. W. (1985). Personality and individual differences: A natural science approach. New York: Plenum.

Everitt, B. S. (1993). Cluster analysis (3rd ed.). New York: Wiley.

Gordon, A. D. (1987). A review of hierarchical classification. Journal of the Royal Statistical Society 150, 119-137.

Lafreniere, K. D., \& Cramer, K. M. (2006). Examining reversal theory measures in relation to NEO personality dimensions and consideration of future consequences. Personality and Individual Differences, 40, 1387-1397.

McDermott, M. R. (1988). Measuring rebelliousness: The development of the Negativism Dominance Scale. In M. J. Apter, J. H. Kerr, and M. P. Cowles (Eds.), Progress in Reversal Theory (pp. 297-312). Amsterdam: Elsevier.

Nunnally, J. C., \& Bernstein, I. R. (1994). Psychometric theory (3rd ed.). New York: McGraw-Hill.

O’Connell, K. A., \& Brooks, E. (1997). Resisting urges and adopting new behaviors. In S. Svebak and M. J. Apter (Eds.), Stress and health: A reversal theory perspective (pp. 157-171). Washington, DC: Taylor \& Francis.

O'Connell, K. A., \& Calhoun, J. E. (2001). The Telic/Paratelic State Instrument (T/PSI): Validating a reversal theory measure. Personality and Individual Differences, 30, 193-204.

Robins, R. W., Hendin, H. M., \& Trzesniewski, K. H. (2001). Measuring global self-esteem: Construct validation of a single-item measure and the Rosenberg Self-Esteem scale. Personality and Social Psychology Bulletin, 27, 151-161.

Robins, R. W., Trzesniewski, K. H., Tracy, J. L., Gosling, S. D., \& Potter, J. (2002). Self-esteem across the lifespan. Psychology and Aging, 17, 423-434.

Sartori, J. (2004). A reversal theory approach to predicting and explaining university students' attitudes and use of marijuana. Unpublished doctoral dissertation, University of Windsor, Windsor, ON, Canada.

Svebak, S., \& Murgatroyd, S. (1985). Metamotivational dominance: A multimethod validation of reversal theory constructs. Journal of Personality and Social Psychology, 48, 107-116. 